\title{
KANTOROVICH PROBLEMS UNDER YOUNG TYPE CONSTRAINTS
}

\author{
Flavia-Corina Mitroi-SymeOnidis And DANIEL AlEXANDRU ION
}

Abstract. We explain how the Hermite-Hadamard inequality agrees with the primal MongeKantorovich problems. We also focus onto a particular case of the dual Kantorovich problem, by considering a version significantly affected by some Young type constraints.

Mathematics subject classification (2010): 49N15, 82C70, 28A35, 90B06, 26A51.

Keywords and phrases: Cost function, Monge-Kantorovich problem, duality, Kantorovich potential, cost convexity, Young's inequality, Hermite-Hadamard inequality.

\section{REFERENCES}

[1] Y. Brenier, U. Frisch, M. Hénon, G. Loeper, S. Matarrese, R. Mohayaee, A. SOBOLEVSKIĬ, Reconstruction of the early Universe as a convex optimization problem, Mon. Not. R. Astron. Soc., 346 (2003), 501-524.

[2] G. Buttazzo, Three optimization problems in mass transportation theory. Nonsmooth mechanics and analysis, 13-23, Adv. Mech. Math., 12, Springer, New York, 2006.

[3] F.-C. Mitroi, D. A. Ion, Structural results on convexity relative to cost functions, Aeq. Math., 85, 1 (2013), 119-130. DOI: 10.1007/s00010-012-0129-y

[4] F.-C. Mitroi, C. P. Niculescu, An extension of Young's inequality, Abs. Appl. Anal., Article ID 162049, doi:10.1155/2011/162049

[5] C. P. Niculescu, J. E. PeČARIĆ, The equivalence of Chebyshev's inequality to the HermiteHadamard inequality, Math. Reports 12 (62), 2 (2010), 145-156.

[6] C. Villani, Topics in optimal transportation, Grad. Stud. Math. 58, American Mathematical Society, Providence, RI, 2003.

[7] C. Villani, Transport optimal de mesure: coup de neuf pour un très vieux problème, Images des mathématiques, 2004, pp. 114-119.

[8] C. Villani, Optimal Transport. Old and New, Series: Grundlehren der mathematischen Wissenschaften, Vol. 338, 2009.

[9] A. Witkowski, On Young's inequality, J. Ineq. Pure and Appl. Math., 7 (2006), Issue 5, article 164. 